\title{
Student's Corner-5
}

\author{
Jayaraman Balachander ${ }^{1}$ \\ ${ }^{1}$ Department of Cardiology, Jawaharlal Institute of Post-Graduate \\ Medical Education and Research, Puducherry, India \\ Ind J Car Dis Wom 2021;6:216-220.
}

\section{Q1) Both Left Ventricle (LV) $S_{3}$ and Pericardial Knock are Filling Sounds but Why Pericardial Knock Does Not Change With Respiration?}

Answer
A. The $\mathrm{LV} \mathrm{S}_{3}$
Expiration can make the $S_{3}$ louder by the following:

1. Increasing blood flow out of the lungs into the left atrium (LA) and LV.

2. The stethoscope coming closer to the chest wall.

3. The apex beat coming out of the ribs in expiration and thus even $S_{3}$ can be palpated by this phase.

4. The $S_{3}$ is very sensitive to the proximity of the stethoscope to the apex beat, since it is a low-pitched sound.

5. When the bell of the stethoscope is applied very lightly to the apical region and the patient is turned to the left lateral decubitus position, the $\mathrm{LV} \mathrm{S}_{3}$ is heard particularly during expiration.

\section{B. The pericardial knock}

1. The ventricle is restricted to the extent in which it can expand.

2. This is compensated by very early diastolic filling.

3. There is also an excessive catecholamine surge in the LV.

4. Further increase in venous return to the right atrium (RA) on inspiration can only cause the filling pressure to increase, as the right ventricle (RV) is unable to accommodate this increase.

5. This increase of the RA pressure and the inability of the RV to accommodate this rise results in rise of jugular venous pressure (JVP) or failure of the JVP to fall on inspiration, producing the Kussmaul's sign.
Address for correspondence Dr. Jayaraman Balachander, Department of Cardiology and Medical Supdt (Retd), Jawaharlal Institute of PostGraduate Medical Education and Research, Puducherry 605006, India (e-mail: balachanderjayaraman@gmail.com).

6. During expiration, the filling pressure of the RA is still high; hence, the filling occurs in the same way to the RV, causing no change in the pericardial knock.

7. However, the pericardial knock is sensitive to changes in blood volume. Diuretics which decrease the RA filling pressure can eliminate the pericardial knock.

\section{Q2) Mechanism of Inconstant Pulmonary Ejection Click}

Answer

Inconstant pulmonary ejection clicks are heard in valvular pulmonary stenosis (PS).

Inconstant pulmonary ejection clicks are heard in valvular PS as evidenced by the following:

1. Their absence in pure infundibular stenosis.

2. Absence if the valve is dysplastic.

3. Occurrence at the peak of the pulmonary valve opening, as seen in echophonocardiography.

The valvular click in PS tends to disappear on inspiration

\section{Mechanism}

1. The sudden upward movement of a dome-shaped pulmonary valve produces the sound.

2. If the valve is already domed or near domed position when the RV contracts, there will be only a soft sound.

3. In valvular PS, the RA has a higher pressure, usually around $10 \mathrm{~mm} \mathrm{Hg}$ at end diastole ("a" wave)

4. In inspiration at end diastole, the contraction of the RA causes the right ventricular end-diastolic pressure (RVEDP) $10 \mathrm{~mm} \mathrm{Hg}$ to rise above the pulmonary artery (PA) pressure. The PA end-diastolic pressure in PS is only $9 \mathrm{~mm} \mathrm{Hg}$
DOI https://doi.org/ 10.1055/s-0041-1736524. ISSN 2455-7854. (c) 2021. Women in Cardiology and Related Sciences. All rights reserved.

This is an open access article published by Thieme under the terms of the Creative Commons Attribution-NonDerivative-NonCommercial-License, permitting copying and reproduction so long as the original work is given appropriate credit. Contents may not be used for commercial purposes, or adapted, remixed, transformed or built upon. (https://creativecommons.org/ licenses/by-nc-nd/4.0/)

Thieme Medical and Scientific Publishers Pvt. Ltd., A-12, 2nd Floor, Sector 2, Noida-201301 UP, India 
5. Thus, the pulmonary valve has already moved to a domed position at the end of diastole and hence will be soft or could disappear.

6. On expiration, the RVEDP falls, and the pulmonary valve is in the down position at the beginning of RV systole.

7. Now, ventricular contraction can balloon the pulmonary valve and dome upward, causing a click

8. This is the only right-sided event that decreases with inspiration.

9. However, in severe PS, the ejection sound may merge with the first heart sound

10. In this case, the clue to the presence of the pulmonary ejection click is as follows:

A. The loud first heart sound in the second left intercostal space.

B. Increasing intensity of the first heart sound with expiration in the second left intercostal space.

\section{Q3) Mechanism of Various Ejection Clicks, How to Differentiate between Various Types of Ejection Clicks, and their Relation with S1?}

\section{Answer}

Ejection clicks are high-pitched sounds that occur at the moment of maximal opening of the aortic or pulmonary valves.

They are heard just after the first heart sound. The sounds occur in the presence of a dilated aorta or pulmonary artery or in the presence of a bicuspid or flexible stenotic aortic or pulmonary valve.

Ejection clicks may also be called ejection sounds.

The diastolic correlate of the ejection click is the opening snap, which occurs at maximal opening of a flexibly stenotic mitral or tricuspid valve.

The various ejection clicks are as follows:

A. Pulmonary.

B. Aortic.

C. Other clicks occurring during the period of ejection.

1. Pulmonary

- Pulmonary valvular ejection click (in constant ejection click).

- Pulmonary vascular ejection click (constant ejection click).

- The pulmonary valvular ejection click (in constant ejection click).

\section{Mechanism (-Fig. 1):}

a. The pulmonary ejection click is produced at the time the pulmonary valve domes in systole in PS. The interval between the first heart sound $S_{1}$ and the occurrence of the pulmonary ejection click varies according to the severity of the PS.

b. These ejection clicks are also heard at the upper left sternal border: with mild or moderate stenosis, there is respiratory variation. In contrast to other right-sided acoustic events (e.g., the murmur of tricuspid regurgitation), the ejection click introducing the murmur of pulmonary stenosis becomes softer during inspiration. Pulmonary stenosis evokes hypertrophy of the right ventricle, stiffening that chamber. When blood is drawn into the right ventricle during inspiration, the thickened right ventricle does not distend normally, so pressure in that chamber rises, and the pulmonary valve is partially opened. Because the pulmonary valve is partially open when the right ventricle contracts, its excursion is less, and the ejection click associated with its maximal opening is softer and earlier

c. With severe valvular pulmonary stenosis, the right ventricle may be so stiff and atrial contraction so vigorous that right atrial contraction actually opens the pulmonary valve completely and produces a click in late diastole.

d. When a phonocardiogram is recorded along with the pulmonary arterial pressure tracing, the ejection click associated with pulmonary stenosis may be seen to correspond with a notch in the upstroke of the pulmonary arterial pressure tracing; this low-frequency mechanical event also occurs at the maximal opening of the pulmonary valve.

e. Systolic ejection clicks occur in early systole and may result from either the abrupt opening of the semilunar valves or the rapid distention of the proximal aorta or pulmonary artery at the onset of ejection. In fact, it is frequently difficult even to separate a systolic ejection click from a split first heart sound or an atrial gallop sound $\left(\mathrm{S}_{4}\right)$ followed closely by $\mathrm{S}_{1}$.

f. Additional information on the relation of valvular events to the principal components of the first heart sound $\left(S_{1}\right)$, combined echocardiograms and phonocardiograms can be recorded

g. A precise relation can be found between completion of closure of the atrioventricular $(\mathrm{A}-\mathrm{V})$ valves manifested in the echocardiogram and the high-frequency components of $\mathrm{S}_{1}\left(\mathrm{M}_{1}\right.$ and $\left.\mathrm{T}_{1}\right)$.

h. The average time from the $\mathrm{Q}$ wave of the electrocardiogram to $\mathrm{M}_{1}$ is usually $0.06 \pm 0.003$ second and the $\mathrm{Q}-\mathrm{T}_{1}$ interval is usually $0.09 \pm 0.002$ second.

i. In pulmonary stenosis, the ejection sound occurs $0.10 \pm 0.003$ second from the $Q$ wave.

$\mathrm{j}$. The ejection sounds of aortic and pulmonary origin coincide exactly with the achievement of a fully opened position of the respective semilunar valve.

k. $\mathrm{M}_{1}, \mathrm{~T}_{1}$, and the ejection sounds occur in association with closing or opening of valves with consequent sudden deceleration or acceleration of a column of blood which, in turn, results in vibrations of the cardio-hemic system.

1. The pulmonary ejection click can occur before the QRS at the end of diastole if the RVEDP is higher than the pulmonary artery diastolic pressure due to atrial systole. It can even occur in early diastole at the peak of early rapid filling if the RV pressure exceeds PA pressure at that time (premature opening of the pulmonary valve). 


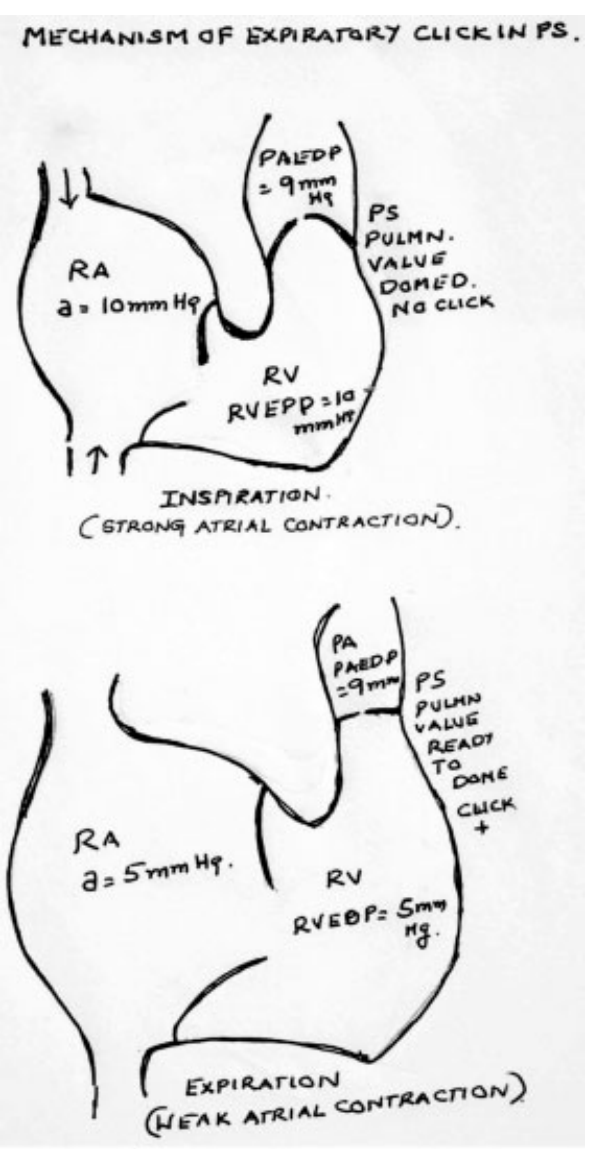

Fig. 1 Mechanism of expiratory click in PS Cited.

$\mathrm{m}$. The RV isovolumic contraction time and the Q-click interval shortens as PS becomes more and more severe because of the following:

1) The rate of RV pressure rise becomes faster.

2) The pulmonary diastolic pressure becomes lower.

3 ) If the stenosis is very severe, the ejection click may merge with $\mathrm{S}_{1}$.

1. The pulmonary vascular ejection click (constant ejection click)

This may occur in

1) Pulmonary arterial hypertension (PAH).

2) Idiopathic dilatation of pulmonary artery.

1). PAH: The high pressure in the pulmonary artery may cause a dilated pulmonary artery at the root, which stretches and tightens the valve ring and cusps which, in turn, produces the click. Echophonocardiography has clearly demonstrated that the click occurs at the peak of the opening of the pulmonary artery. This sound differs from the ejection click in PS, in that it is heard at the third left intercostal space and is not affected by respiration (constant ejection click). This is usually a feature of Eisenmenger syndrome due to ventricular septal defect (VSD) or atrial septal defect (ASD). It could be heard also in primary PAH. This ejection click is usually heard close to the first heart sound

2). Idiopathic dilatation of pulmonary artery (IDPA): This is due to jerky sudden expansion of an abnormal pulmonary artery. This sound is heard far from the first heart sound. This is the reason of occurrence of an occasional click in the absent pulmonary valve syndrome, due to the massive dilatation of proximal pulmonary artery.

3). Sometimes in cases of ASD without pulmonary artery dilatation, ejection clicks, which are vascular, can be heard due to the rapid flow of large amounts of blood across the normal pulmonary valve. If there is an ejection click with a loud ejection systolic murmur in ASD, then ASD with PS can be suspected. The other condition which produces the similar clinical picture is Lutembacher's syndrome where a large flow occurs into a dilated pulmonary trunk.

\section{Left-sided ejection clicks}

1. Left-sided ejection clicks occur in the presence of a dilated aorta or aortic valve abnormalities. When the aortic valve is normal, left-sided ejection clicks seem to be related to dilation of the aortic root, even though the ejection click occurs at maximal opening of the normal aortic valve.

2. Ejection clicks associated with a dilated aortic root are best heard at the aortic area and are poorly transmitted to the apex.

3. Aortic valvular ejection clicks, in contrast, are loudest at the apex. When there is aortic stenosis, the aortic ejection click just precedes the murmur. Ejection clicks occur with flexibly stenotic aortic valves and nonstenotic bicuspid aortic valves.

4. It may be difficult or impossible to distinguish an aortic ejection click from the tricuspid component of a widely split $S_{1}$. The tricuspid component of $S_{1}$ is best heard at the left lower sternal border and becomes louder with inspiration.

5. The combination of $S_{4}$ and $S_{1}$ may also be mistaken for an $\mathrm{S}_{1}$ ejection click.

6. The $S_{4}$ (atrial gallop) is best heard with the bell lightly applied to the cardiac apex and is associated with a presystolic apical impulse.

7. Like right-sided ejection clicks, the aortic ejection click may correspond to a notch in the upstroke of the aortic pressure tracing or the carotid pulse tracing This notch apparently results from maximal opening and abrupt deceleration of the flexibly stenotic aortic valve.

8. When the aortic valve is immobilized by heavy calcification, the sudden deceleration of cusp motion cannot occur, so there is no ejection sound; the aortic component of the second heart sound is soft or absent. 
9. When an ejection click is associated with a nonstenotic bicuspid aortic valve, the ejection click is loud and widely separated from the first heart sound.

10. Other causes of systolic clicks have been described as follows:
A. Mitral valve prolapse
B. The pseudoejection sound of idiopathic hypertrophic subaortic stenosis.
C. Aortic dissection.
D. Ventricular septal aneurysm.

\section{Conclusion}

Correct identification of an ejection click prevents confusion with other heart sounds, localizes ventricular outflow obstruction to the valvular level, or suggests the presence of a bicuspid aortic valve or dilation of the aorta or pulmonary artery. ${ }^{1}$

\section{Reference}

1 Nemani Lalita, Pechetty Ramya. Additional Heart Sounds -Part 2 (Clicks, Opening Snap and More). Indian J Cardiovasc Dis Women WINCARS 2020;5(04):351-363 
